\title{
Camel coccidiosis caused by Isospora orlovi in the United Arab Emirates
}

\author{
J. Kinne ${ }^{1}$, M. Ali ${ }^{2}$ and U. Wernery ${ }^{1}$ \\ ${ }^{1}$ Central Veterinary Research Laboratory, P.O. Box 597, Dubai, UAE \\ ${ }^{2}$ Camel Reproduction Laboratory, P.O. Box 9229, Nakhlee, Dubai, UAE
}

\begin{abstract}
Between January and March 2001, a total of 22 dromedary calf carcasses ftom Dubai area were submitted for post-mortem examination. Eight of these calves ( 4 to 8 weeks old) came from 2 farms and showed diarrhoea between 2 and 5 days with no response to any treatment. Gross pathological examination of these calves revealed a severe diphtheroid to haemorrhagic colitis. Massive numbers of small coccidian-stages and some eosinophilic granulocytes were detected in the lamina propria by histopathological studies. It was considered that these stages belong to an Isospora spp., because typical oocysts containing 2 sporocysts with 4 sporozoites each were found in colon smears and faecal samples. Two Isospora spp. are described in camels: I orlovi and I cameli. However, I orlovi is thought to be an avian form, accidentally ingested. The oocysts of our Isospora spp. seem to be very similar to $I$ orlovi. The observed colitis associated with a massive invasion of Isospora-organisms suggests that this species is much more pathogenic than reported in the literature.
\end{abstract}

Keyword: coccidiosis, diarrhoea, calves, camels.

\section{Introduction}

Six species of intestinal coccidian parasites have been described for old World camelids (Levine and Ivens, 1970. Levine, 1985). They are five species of Eimeria and one species of Isospora (I orlovi). However, there may be other Isospora species e.g. I cameli (Zigankoff, 1950. Kaufmann, 1996). There are numerous reports about coccidiosis in camels in the literature. However, severe coccidiosis causing enteritis and a mortality rate up to $10 \%$ in young camels have only been reported in a few cases (Hamanchandran et al., 1968. Gruvel and Grab ER, 1969. Chineme, 1980. Levine, 1985. Hussein et al., 1987). On the other hand, many reports concerning the presence of coccidian oocysts are based on investigations of faecal samples of healthy camels. Gruvell and Graber (1969). found only 14 out of 204 faecal samples to be positive forcoccidian oocysts. Mirza et al. (1976). diagnosed $86 \%$ of faecal samples to be positive for $\mathrm{E}$. dromedarii and $\mathrm{E}$. cameli oocysts in Iraq. Dubey and Pande (1964). identified E. rajasthani, E. dromedarii and E. noelleri oocysts in faecal samples from 45 healthy Indian camel calves. Gill (1976) found oocysts of E. cameli, E. dromedarii, E. pellerdyi and E. bactriani in $24 \%$ faecal samples from dromedaries in India. Y Agoub (1989) detected $17.4 \%$ of a total of 230 faecal samples submitted from Sudanese camels to be positive for coccidian oocysts. All these reports regarding camel coccidiosis are dealing with Eimeria spp. However, there are only few reports of Isospora-infections in camels. Raisanghani et al. (1987) described one case in a six month old camel calf showing diarrhoea and abdominal pain. Faecal examination revealed 2000 Isospora oocysts per gram faeces. Size and shape of the oocysts were similar to I orlovi. However, the authors did not identify the parasite. I orlovi (Zigankoff, 1950) is thought by Pellerdy (1965) to be an avian form, accidentally ingested. The pathogenic role of the two Isospora spp., I orlovi and I cameli is unknown (Kaufmann, 1996).

\section{Materials and Methods}

Between January and March 2001, a total of 22 camel calf carcasses from Dubai area were submitted for post-mortem examination. Eight of these calves (4 to 8 
week-old) came from 2 farms and showed diarrhoea between 2 and 5 days with no response to any treatment. All autopsies were performed within 1 to 5 hours after death. Pieces of intestine, liver, spleen and lymph nodes were taken for microbiological investigations using routine methods. The intestinal samples were also tested for the growth of anaerobes. For histopathological investigation, small pieces of all organs including compartment 1 and stomach, as well as muscles and body lymph nodes were taken. These samples were fixed for 24 hours in $10 \%$ buffered Formalin. Five !lm thin sections were cut and stained with haematoxilin and eosin.

Faecal samples from each camel calf were collected and tested with the flotation method. Direct smears were also prepared and examined. Twenty oocysts were measured under the microscope and average lengths and widths were calculated.

\section{Results}

Between January and March 2001, a new of camel calf disease occurred in the Dubai area. The calves on two farms showed diarrhoea between 2 and 5 days with no response to any treatment. No changes in feeding practice were observed. The mothers, which were kept in large pens, received hay ad libitum and small amounts of pellets. When the calves became sick, they were kept in smaller pens together with their mothers, which never showed any disease.

Gross pathological examination of eight calves from these 2 farms revealed severe diphtheroid to haemorrhagic colitis (Fig. 1). Massive numbers of small coccidian-stages were detected in the lamina propria of the colon by histopathological studies. Histological sections showed destruction and disorganisation of the mucosa together with haemorrhages and infiltration of inflammatory cells, mainly eosinophils and macrophages. Numerous coccidian stages (zygotes and oocysts) were located in the mucosa ofthe colon (Fig. 2), but not in the small intestine. These oocysts, containing 2 sporocysts with 4 sporozoites each (Fig. 3) were also found in colon smears and faecal samples. Most of the oocysts were sporulated at the time of investigation.

The oocysts were ellipsoidal to ovoid, figure 8-shaped, $30-33 \times 18-21 /-\mathrm{Lm}$ with a smooth, 2-layer wall about $1 /$-Lm thick; without a micropyle, polar granule, or residuum. The two sporocysts per oocyst were ellipsoidal with a size of 10-15 x 1719 /-Lm containing four elongated ellipsoidal sporozoites 4-6 x 11 -13/-Lm in size. Microbiological investigations including anaerobic culture did not detect pathogenic bacteria.

\section{Discussion}

Severe coccidiosis causing enteritis and a mortality rate up to $10 \%$ in young camels have only been reported in a few cases (Hamanchadran et al., 1968. Gruvel and Grab ER, 1969. Chineme, 1980; Levine, 1985; Hussein, 1989. Kinne and Wernery, 1997). The coccidian species associated with disease (coccidiosis) are primarily E. cameli and E. dromedarii, but also E. rajasthani seems to be pathogenic (Hussein et al., 1987). All these species are belonging to the Eimeria-genus.

Between January and March 2001, a new camel calf disease occurred in the Dubai area. The calves on two farms showed diarrhoea between 2 and 5 days with no response to any treatment. Eight of these calves ( 4 to 8 weeks old) were submitted for post-mortem examination. Gross pathological examination of these eight calves revealed severe diphtheroid to haemorrhagic colitis. Massive numbers of small coccidian-stages were detected in the lamina propria by histopathological studies. Eimeria spp. are distinguished from Isospora spp. by the sporulated oocysts. 
Eimeria spp. contain 4 sporocysts with 2 sporozoites each, whereas Isospora spp. have 2 sporocysts with 4 sporozoites each (Levine, 1985). It was considered that the coccidian stages in our cases belong to an Isospora spp., because typical oocysts (containing 2 sporocysts with 4 sporozoites each) were found in colon smears and faecal samples.

The oocysts of $I$. orlovi are described as ellipsoidal, ovoid, piriform, 'to figure 8shaped, 27-35 x 15-20 flm with a smooth, 2-layer wall about 1 um thick without a micropyle, polar granule, or residuum. The sporocysts are ellipsoidal to ovoid (13-15 flm in diameter) with a residuum and without Stieda body. The sporozoites are elongated ellipsoidal, 7-10 x 4-6 flm (Levine, 1985; Pellerdy, 1965). As the oocysts in our cases resembled I orlovi, it is believed that the outbreak was caused by $I$ orlovi.

It is worthwhile to note, that around 50 $\%$ of the oocysts were already sporulated in the faecal samples at time of investigation. In our laboratory it usually takes 6 to 24 hours to examine the slides after sampling. This might be long enough for oocysts sporulation. The high number of sporulated oocysts in the faeces may be a reason, that I orlovi is thought by Pellerdy (1965). to be an avian form, accidentally ingested. As they are multiplying in the colon mucosa causing severe colitis (and no pathogenic bacteria were detected), our Isospora spp. is thought to be an original camel pathogen.

The source of infection remained unknown. The calves got only milk form their mothers and may have ingested also some hay. Since copper deficiency was diagnosed in dromedary calves and their dams in the UAE (Wernery et al. 2000) the calves may have tried to compensate a copper deficiency by ingesting sand containing Isospora oocysts. Treatment with Baycox ${ }^{\circledR}$ in the recommended dosage was unsuccessful.

\section{Figures}

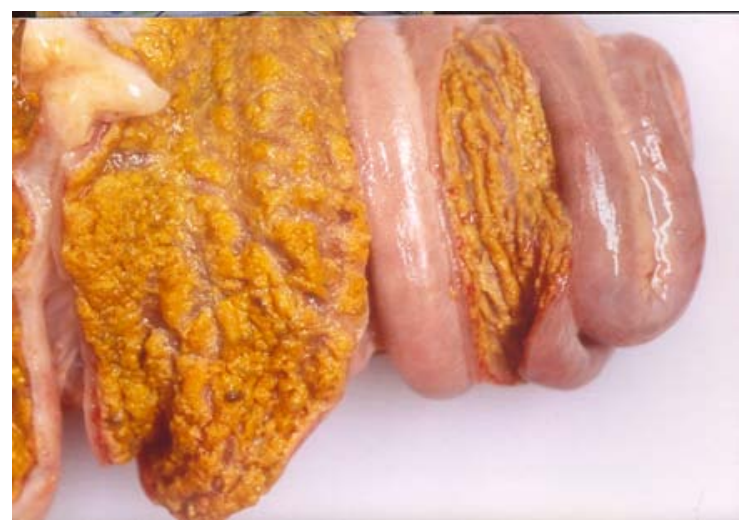

Fig. 1: Severe diphtheroid to haemorrhagic colitis caused by Isospora orlovi.

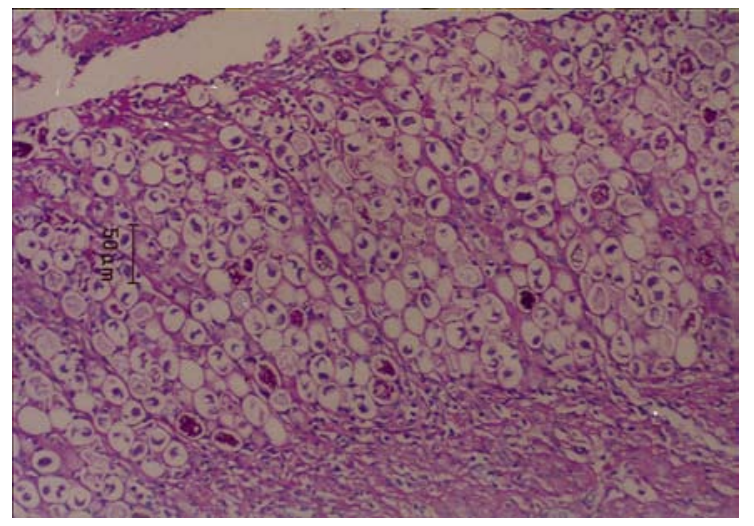

Fig. 2: Coccidian stages (zygotes and oocysts) were located in the mucosa ofthe colon

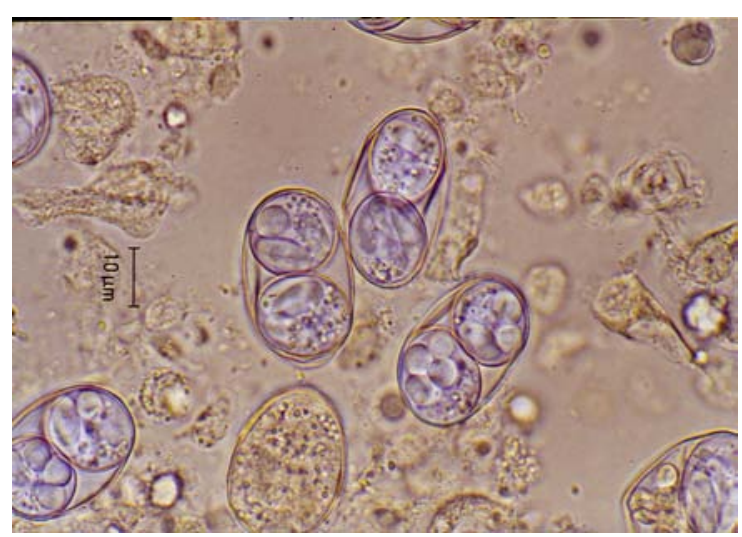

Fig. 3: Oocysts containing 2 sporocysts with 4 sporozoites each 


\section{References}

Chineme, C.N., 1980. A case report of coccidiosis caused by Eimeria cameli in a camel (Camelus dromedarius) in Nigeria. J. Wildlife Dis.1Q, 377 - 380 .

Dubey, U.P., R.P. Pande, 1964. On Eimerian oocysts recovered from Indian Camel (Camelus dromedarius). Indian J. vet. Sci. 34, $28-34$

Gill, H.S. 1976. Incidence of Eimeria and Infundibulorium in camel. Indian Vet. J..TI.., 897-898. Gruvell, J., Grab ER, M., 1969. Coccidia and Coccidiosis. 2nd. ed. Akademia Kiado, Budapest, 698.

Hamanchandran, P.K., S. Ramachnadran, T.P. Joshi, 1968. An outbreak of hemorrhagic gastro enteritis in camels. Ann. de Paras 5 -14.

Hussein, H.S., A.A. Kasim, W.A. Sha, YR., 1987. The prevalence and pathology of Eimeria infections in camels in Saudi Arabia. J. Comp. Path. 97,293 - 297.

Kaufmann, J. 1996. Parasitic infections of domestic animals - A diagnostic manual, Birkhiiuser Verlag, Basel, Boston, Berlin.

Kinne, J. and U. Wernery, 1997. Severe outbreak of camel coccidiosis in the United Arab Emirates. J. Camel Pract. and Res. ::L 261-265.
Levine, N.D. 1985. Veterinary Protozoology, $1^{\text {st }}$ ed. Iowa State University Press, Ames. 202.

Levine, N.D. and V. Ivens. 1970. III Biology Monograph Series No. 44, Urbana, University of Illinois Press.

Mirza, M.Y, AS Al Raw, A.Y 1976. Bulletin of the Biological Research Center, Baghdad, 1, 24.

Pellerdy, L.P. 1965. Coccidia and Coccidiosis. Akademiai Kiado, Publishing House of the Hungarian Academy of Sciences, Budapest, 510-516.

Raisanghani, P.M., G.S. Manohar, and J.S. Y Ada V, 1987. Isospora infection in the Indian camel (Camelus dromedarius). Indianj. of Parasitology. 11, 93-94.

Wernery, U., M. Ali, J. Kinne, A. A. Abraham and Renate Wernery, 2000. Copper deficiency: a predisposing factor to septicaemia in dromedary calves. 2nd International Camelid Conference, Almaty, Kazakhstan. 8. -12.9.2000.

Y Agoub, LA. 1989. Coccidiosis in Sudanese Camels (Camelus dromedarius): 1 - First record and description of Eimeria spp. harboured by camels in the eastern region of Sudan. J. Protozool. $36,422-423$.

Zigankoff, A.A., 1950. Contribution to the knowledge of coccidian trom the camel. Izv. Kazah. SSR. Ser. Par. 